\title{
Simulation Research on the Formation Behavior of a Compound College Students' Entrepreneurship Team Based on NetLogo
}

\author{
Mingxuan Qin*, Liangliang Chen, Na Jing, Qiuxing Chen \\ School of Entrepreneurship, Wuhan University of Technology, Wuhan, 430070, China \\ ${ }^{*}$ Corresponding author
}

\begin{abstract}
At present, there is still a lot of room for development and improvement in the research methods and research results of the formation of college students' entrepreneurship teams. This paper analyzes some influencing factors of forming a compound college students' entrepreneurship team such as students' knowledge structure, team cooperation ability and interdisciplinary communication environment. This paper models the complex behavior of building a compound entrepreneurial team for college students, and uses netlogo for simulation research. The research found that the stronger the students' professional knowledge ability, the higher the team cooperation ability, and the more opportunities for interdisciplinary communication with other students, the higher the efficiency and quality of building a compound entrepreneurship team for college students.
\end{abstract}

Keywords: College student, Entrepreneurial teams, Simulation

\section{复合型大学生创业团队组建行为的NetLogo仿真研究}

\author{
秦鸣轩*, 陈亮亮, 荆娜, 陈求星
}

武汉理工大学创业学院, 武汉, 中国

“通讯作者

\section{中文摘要}

对于大学生创业团队组建行为的研究, 目前在研究方法和研究成果上还有很多可以发展提高的空间。 本文分析了在大学生组建创业团队过程中, 学生的知识结构、团队合作能力、跨学科交流的环境对于 组建复合型、多能力的创业团队的影响。对组建复合型大学生创业团队的复杂行为进行建模, 并利 用 NetLogo 进行仿真研究。研究发现, 学生的专业知识能力越强、团队合作能力越高、与其他专业学 生进行跨学科交流的机会越多，那么组建成复合型大学生创业团队的效率和质量就越高。

关键词：大学生；创业团队；仿真

\section{1. 引言}

大学生作为具有较高知识能力和创新能力的年轻 群体，在中国提倡 “大众创业、万众创新” 的政策推动 下, 成为了中国创新创业领域的重要参与者。大学生参 与创新创业活动的优势在于他们拥有一定的技术创新 能力、较为完备的知识结构和强烈的创新创业动机。然 而, 创业是一项复杂、高风险的行为, 大学生在很多方 面还达不到创业的条件。因此, 就需要具有创业意愿的
大学生们联合起来, 利用自身优势, 组建成复合型的创 业团队一一即由来自不同专业领域, 拥有不同知识结构 和能力的大学生组成的创业团队, 投身于创业活动。

\section{2. 影响复合型大学生创业团队组建的因素}

通过阅读研究有关大学生创业行为和大学生创业 团队组建的文献, 并且访谈10名较为成功的大学生创业 者和5名创新创业教育的专家。我们确定了三种影响大 学生组建复合型创业团队的因素。 
(1) 学生的知识能力

综合各类学者近年来对我国大学生创业的研究成 果, 可以将大学生创业模式分为复制型创业、模仿型创 业和创新型创业。创新型创业包括技术驱动型创业和创 意驱动型创业, 是推动国家科技创新、经济转型升级和 创造就业机会的主要驱动力。[1]在中国经济进入新形 势的背景之下，国家更加鼓励大学生利用自己的知识优 势, 更多的从事创新型创业。这需要创业团队拥有更加 完备和高深的专业知识能力。

(2) 团队合作的能力

当今社会是一个强调精益化生产、分工合作的社 会。一个大学生创业团队要成功创业就不能完全脱离这 样分工日益精细的社会。一个人也许可以指凭借自己 的能力在某个方面取得令自己满意的成果, 但一群不同 能力的人把各自特有的能力有效结合并发挥出来, 也许 会获得令人吃惊的成果。[2]一个合作默契和谐的创业 才队，才能有机会取得创业的成功。

(3) 跨学科交流的环境

科学上新理论、新发明的产生以及新的工程技术的 出现, 经常是在学科的边缘或交叉点上。从高度分化走 向交叉融合已成为学科发展的客观规律。[3] 学科发展 和技术创新强调学科交叉, 大学生创业也是如此。跨 学科具有综合创造性、互补性和群发性等特征, 能够有 效实现不同学科间的交叉融合, 有利于培养学生的创造 思维和创新能力。 [4]一个创业团队如果具有跨学科的 特点, 这个团队将会具有多元化、多能力的优势, 在创 新过程和创业项目实际运营的过程中, 与单一化学科的 团队相比, 将具有解决多种问题的能力。

\section{3. 复合型大学生创业团队组建行为建模}

\section{1. 仿真工具选择及介绍}

复合型大学生创业团队的组建行为具有复杂性, 涉 及多种影响因素和多个主体的交互, 因此, 利用计算机 仿真, 尤其是利用代理人基模型（agent-based mode1, ABM）进行研究, 具有较好地模拟现实情境, 又能尽量 避免主观因素影响的优势 [5]。有很多的计算机仿真系 统可以用来模拟团队组建行为, 例如 Swarm、Repast、 MASON 和 NetLogo等。相比较而言, NetLogo 有自己独 特的优势: 它不仅可基本实现各种复杂的社会现象仿 真, 而且能提供友好的界面保证易学性和易用性 [6]。 NetLogo 仿真工具, 其模型主体分为海龟 (Turtles)、 瓦片 (Patches)、观察者 (Observer) 三类. Turtles 是 行为者, 可以在网格环境 (Patches) 中移动; Patches 构成网格环境, 作为 Turtles 的活动空间. Observer 是 主要命令的发布和协调者, 运行状态的观察者. Observer 的主要任务是在设定了主体与主体、主体与 环境之间的行为规则后, 在 Netlogo 平台中自行设置
环境和行为者以及一些系统环境变量, 从而让行为者和 环境在这些设定规则下运行出复杂的结果。

\section{2. 模型介绍}

为了研究复合型大学生创业团队的组建机制, 根据 实际情况、研究要求和前期归纳总结的影响因素。我们 利用NetLogo设计构建了一个多主体模型. 即在一个二 维的虚拟世界中分布着两种不同性质的主体: “理工专 业学生”、“经济管理专业学生”。这两种类型的主体被 分别划分在两个不同的区域里面, 代表实际中理工科专 业与人文经管专业学生接触交流的限制。每位主体在自 己所划分的区域移动，不同区域内的主体拥有一定的概 率走出自己的区域，进入公共区域。每位主体都拥有三 种变量： A技术创新值、B商业创新值, C团队协作值。 理工科学生 $A$ 值较高, B值较低; 经管专业学生 $A$ 值较低、 B值较高, 每位主体都有机会与其他主体相遇交流, 获 得 $A$ 值与 $B$ 值的增长。当相遇的主体 $A$ 值、 $B$ 值、C 值达到 标准时，他们就会形成复合型的创业团队。通过观察组 建成功的复合型创业团队数量和所用时间, 我们可以得 到不同因素对于大学生组建复合型创业团队的影响效 果。

\section{3. 仿真实验}

本研究基于以上模型, 通过判断 “已组建的团队” 的数量变化及达成所要求的的团队数量的时间来研究 影响大学生复合型创业团队组建的因素如何对其行为 施加影响, 并直观地观察了大学生创业者组建复合型创 业团队的过程. 文章将以 Windows 下的 NetLogo 6.04 仿真平台建立模型，主要变量如下：

1）主体初始的A、B、C值：理工科专业学生 A值在 80-120之间随机； B值在 20-60之间随机；C值在 10-30之 间随机。经济管理专业学生A值在 20-60之间随机; B值 在80-120之间随机; C值在 10-30之间随机。当两个主体 相遇时, 他们会将相互的A、B值进行比较, 分别取两者 之间最大的一个, 实现 $A 、 B$ 值的增长。

2）主体进入公共区域的概率：不同类型的主体拥 有一定概率离开自己所在的区域, 前往公共区域, 象征 着跨学科交流。主体进入公共区域的概率为 $0-1$ 。

3) 组成创业团队的阈值: 当两个主体相遇时, 在 完成 $A 、 B$ 值的增长之后, 若个主体的 $A 、 B 、 C$ 值达到设 定的阈值, 则视为两者组建成一支复合型创业团队。若 两个主体的 $A$ 值和大于 $100-200$ 、B值大于 100-200、且两 者C值都不小于 $0-20$, 则达到要求。

模型开始运行之后, 每个时间步内, 每个学生在环 境内移动一次, 若学生相遇, 则进行交互, 实现 $\mathrm{A} 、 \mathrm{~B}$ 值 的增长。结束一个时间步后, 检查是否满足组成团队的 要求, 若满足, 则组为团队, 且不再与其他主体交互, 若不满足，则进行下一个时间步。当模型内的已组建团 
队的数量达到目标值或时间步达到最大值时, 模型停止 运行。

\section{4. 实验结果及分析}

\section{1. 实验结果}

为了体现实验的严谨性和真实性, 每种条件下我们 都进行了 10 次实验, 通过取平均数的方式进行比较。

表1 实验 1 第一次实验结果

\begin{tabular}{|c|c|c|c|c|c|c|c|c|c|c|c|}
\hline 次数 & 1 & 2 & 3 & 4 & 5 & 6 & 7 & 8 & 9 & 10 & 平均步数 \\
\hline 时间步 & 157 & 170 & 144 & 191 & 171 & 198 & 176 & 195 & 227 & 189 & 181.8 \\
\hline
\end{tabular}

ii) 将进入公共区域的概率的设定为 0.5 , 初始人数 将组建成团队的阈值设置为两个主体的 $A$ 值和大于 200 , 设定为两类学生各 60 个, 组建团队的目标个数为 16 支。

表2 实验1第二次实验结果

\begin{tabular}{|c|c|c|c|c|c|c|c|c|c|c|c|}
\hline 次数 & 1 & 2 & 3 & 4 & 5 & 6 & 7 & 8 & 9 & 10 & 平均步数 \\
\hline 时间步 & 157 & 170 & 144 & 191 & 171 & 198 & 176 & 195 & 227 & 189 & 181.8 \\
\hline
\end{tabular}

实验 2 : 研究团队合作能力对于组建复合型大学生将组建成团队的阈值设置为两个主体的A值和大于 200 , 创业团队的影响

i) 将进入公共区域的概率的设定为 0.5 , 初始人数 设定为两类学生各 60 个, 组建团队的目标个数为 16 支。

表 3 实验 2 第一次实验结果

\begin{tabular}{|c|c|c|c|c|c|c|c|c|c|c|c|}
\hline 次数 & 1 & 2 & 3 & 4 & 5 & 6 & 7 & 8 & 9 & 10 & 平均步数 \\
\hline 时间步 & 157 & 170 & 144 & 191 & 171 & 198 & 176 & 195 & 227 & 189 & 181.8 \\
\hline
\end{tabular}

ii) 将进入公共区域的概率的设定为 0.5 , 初始人数 将组建成团队的阈值设置为两个主体的 A值和大于 200 , 设定为两类学生各 60 个, 组建团队的目标个数为 16 支。 B值大于 200 , 双方 C值不小于 20 。结果如下（表4）

表4 实验2第二次实验结果

\begin{tabular}{|c|c|c|c|c|c|c|c|c|c|c|c|}
\hline 次数 & 1 & 2 & 3 & 4 & 5 & 6 & 7 & 8 & 9 & 10 & 平均步数 \\
\hline 时间步 & 157 & 170 & 144 & 191 & 171 & 198 & 176 & 195 & 227 & 189 & 181.8 \\
\hline
\end{tabular}

实验 3 : 研究跨学科交流环境对于组建复合型大学 将组建成团队的阈值设置为两个主体的 $A$ 值和大于 200 , 生创业团队的影响

i) 将进入公共区域的概率的设定为 0.6 , 初始人数 设定为两类学生各 60 个, 组建团队的目标个数为 16 支。

\section{表5 实验3第一次实验结果}

\begin{tabular}{|c|c|c|c|c|c|c|c|c|c|c|c|}
\hline 次数 & 1 & 2 & 3 & 4 & 5 & 6 & 7 & 8 & 9 & 10 & 平均步数 \\
\hline 时间步 & 157 & 170 & 144 & 191 & 171 & 198 & 176 & 195 & 227 & 189 & 181.8 \\
\hline
\end{tabular}

ii ）将进入公共区域的概率的设定为 0.8 , 初始人 支。将组建成团队的阈值设置为两个主体的 $A$ 值和大于 数设定为两类学生各 60 个, 组建团队的目标个数为 $16 \quad 200$, B值大于 200 , 双方 C值不小于 20 。结果如下（表6） 表6 实验3第二次实验结果

\begin{tabular}{|c|c|c|c|c|c|c|c|c|c|c|c|}
\hline 次数 & 1 & 2 & 3 & 4 & 5 & 6 & 7 & 8 & 9 & 10 & 平均步数 \\
\hline 时间步 & 157 & 170 & 144 & 191 & 171 & 198 & 176 & 195 & 227 & 189 & 181.8 \\
\hline
\end{tabular}

iii) 将进入公共区域的概率的设定为 0.3 , 初始人 数设定为两类学生各 60 个, 组建团队的目标个数为 16 支。将组建成团队的阈值设置为两个主体的 $A$ 值和大于
200 ，B值大于 200 , 双方C值不小于 20 。结果表明, 都无 法在规定的时间步内（500步）达成目标数量。 


\section{2. 结果分析}

根据实验结果表明，在其他条件不变的情况下，当 组成团队的阈值中 $A$ 值和B值要求越高时, 达成目标团队 人数所花费的时间步就越多。说明, 当团队对于团队成 员的知识能力水平要求越高时, 组建团队就越困难。另 一方面, 当学生的知识能力越强时, 组建团队的效率就 更高。

在其他条件不变的情况下，当组成团队的阈值中 $C$ 值要求越高时, 达成目标团队人数所花费的时间步就越 多。说明, 当团队对于团队成员的团队合作能力要求越 高时, 组建团队就越困难。另一方面, 当学生的团队合 作能力越强时, 就更容易组成创业团队。

在其他条件不变的情况下, 当进入公共区域的概率 越高时, 达成目标团队人数所花费的时间步就越少。说 明, 当学生之间跨学科交流的机会越多, 环境越宽松,

就能更快地达到组成团队的要求, 形成创业团队。当跨 学科交流的机会非常小时, 将会极大地影响组建团队的 效率。

\section{5. 结论}

本文通过进行计算机仿真, 对于大学生组建复合型 创业团队的机制进行了一定的研究。发现学生的知识能 力、团队合作能力和学生之间跨学科交流是影响组建复 合型团队的重要因素。作为大学生, 如果想在创业中有 所建树, 组建一支富有创造力、凝聚力、多能力的团队, 则需要加强知识学习, 丰富知识能力, 提升自己的团队 合作意识和协作能力, 并且具有多元化思维, 乐于与其 他学科的学生进行交流和合作。作为高校, 如果想提升 本学校的创新创业水平, 形成更多有活力的学生创业团 队, 则首先提高科研教学水平, 其次开展创业教育, 激 发学生的创业意识和团队合作能力, 最后, 也要构建一 个跨学科交流的平台与机制, 鼓励学生进行跨学科交 流。

\section{致谢}

本文为本文为国家级大学生创新创业训练计划资 助项目（201910497241）的阶段性成果之一。

\section{REFERENCES}

[1] Jia Bo, Jin Shaorong. Logic and realization path of innovative entrepreneurship of college students in the new normal $[\mathrm{J}]$. School party building and ideological education, 2016 (08): 63-65

[2] Wang Hong, Zheng Zedong, Meng Siqi. On the construction of innovation and entrepreneurship team of college students $[\mathrm{J}]$. Cultural and educational materials, 2017 (30): 157-158
[3] Shao Jin. Two entry points of innovation and Entrepreneurship Education: innovation ability and cross-border ability training -- thinking, exploration and practice of Nanjing University [J]. Teaching of China University, 2016 (12): 40-43

[4] Lin Xiaoling. On the construction of interdisciplinary innovation curriculum system in Colleges and universities from the perspective of innovation and entrepreneurship [J]. University education, 2017 (01): $1-5$

[5] Xu Chunlin. Research on cooperative behavior based on MAS [D]. Yangzhou University, 2007

[6] Lu Yunbo. Research on the relationship between team design variables and team performance [D]. Tongji University, 2007 